\title{
Dynamics of a green high-power tunable external-cavity broad-area GaN diode laser
}

\author{
Chi, Mingjun; Jensen, Ole Bjarlin; Hansen, Anders Kragh; Petersen, Paul Michael
}

Published in:

Optical Society of America. Journal B: Optical Physics

Link to article, DOI:

10.1364/JOSAB.35.000667

Publication date:

2018

Document Version

Peer reviewed version

Link back to DTU Orbit

Citation (APA):

Chi, M., Jensen, O. B., Hansen, A. K., \& Petersen, P. M. (2018). Dynamics of a green high-power tunable external-cavity broad-area GaN diode laser. Optical Society of America. Journal B: Optical Physics, 35(4), 667671. https://doi.org/10.1364/JOSAB.35.000667

\section{General rights}

Copyright and moral rights for the publications made accessible in the public portal are retained by the authors and/or other copyright owners and it is a condition of accessing publications that users recognise and abide by the legal requirements associated with these rights.

- Users may download and print one copy of any publication from the public portal for the purpose of private study or research.

- You may not further distribute the material or use it for any profit-making activity or commercial gain

- You may freely distribute the URL identifying the publication in the public portal 


\title{
Dynamics of a green high-power tunable external- cavity broad-area GaN diode laser
}

\author{
Minguun Chi, ${ }^{*}$ Ole B. Jensen, Anders K. Hansen, and Paul M. Petersen \\ DTU Fotonik, , Department of Photonics Engineering, Technical University of Denmark, Frederiksborgvej 399, P.O. Box 49, DK-4000 Roskilde, \\ Denmark \\ *Corresponding author: mchi@fotonik.dtu.dk
}

Received XX Month XXXX; revised XX Month, XXXX; accepted XX Month XXXX; posted XX Month XXXX (Doc. ID XXXXX); published XX Month XXXX

\begin{abstract}
Although external-cavity feedback with a grating is widely used to achieve tunable high-power narrow-linewidth broad-area diode laser (BAL) system, the dynamics of such system is seldom studied. In this paper, the temporal dynamics of a tunable high-power green external-cavity diode laser system based on a GaN BAL and Littrow externalcavity is investigated experimentally. The regular pulse package oscillation (PPO) is observed just above the threshold. The oscillating period of the pulse package decreases with the increasing injected current. As the current increases further, the pulse package oscillates irregularly, and finally changes to a chaotic state. The PPO is observed, for the first time to our knowledge, in a BAL with an external-cavity grating feedback. (C) 2017 Optical Society of America
\end{abstract}

OCIS codes: (140.5960) Semiconductor lasers; (140.2020) Diode lasers; (190.3100) Instabilities and chaos.

http://dx.doi.org/10.1364/AO.99.099999

\section{INTRODUCTION}

Significant progress has been achieved on GaN-based high power green diode lasers since 2009 [1,2]. Today, green GaN broad-area diode lasers (BALs) with continuous-wave (CW) output power of more than $1 \mathrm{~W}$ are commercially available [3]. The spectral bandwidth of these high-power BALs is around $1 \mathrm{~nm}$, and this broad emission bandwidth limits the application of these devices in many fields, such as laser holographic display, biophotonics [4,5]. External-cavity feedback based on a grating is a commonly used technique to achieve a tunable narrow-bandwidth high-power diode laser system [6-9]. Recently, we demonstrated a highpower tunable diode laser system based on Littrow external-cavity feedback around $515 \mathrm{~nm}$, where an output power of up to $480 \mathrm{~mW}$ and a tunable range of up to $9.2 \mathrm{~nm}$ was achieved, and the spectral bandwidth is around $9 \mathrm{pm}$ with low output power and less than 0.25 nm with high output power [10].

When external-cavity feedback is applied to a diode laser, complex temporal dynamic behaviors will take place. The different dynamic behaviors of BALs have been studied intensively with short-cavity feedback [11-13], long-cavity feedback [14-16], tilt mirror feedback $[17,18]$, and lateral-mode-selected feedback $[19,20]$. The feedback elements in the external-cavity include ordinary mirrors [13-18], phaseconjugate mirror [14], and spatially filtered-mirrors [11,12,19,20]. Different dynamic behaviors, such as self-pulsation [19], low frequency fluctuations (LFFs) $[14,15]$, periodic oscillations $[17,20]$, pulse package oscillation (PPO) $[12,13,20]$ and chaos [16], have been observed in the BALs subjected to different external-cavity feedback.

External-cavity grating feedback is widely used to achieve tunable high-power narrow-bandwidth BAL system. However, the dynamics of such systems have only been studied in a very few cases [21]. The research of the dynamics of BAL systems with external grating feedback is important for both the basic laser physics and the application of such a laser system.

In this paper, we investigate the dynamics of the tunable high-power green diode laser system developed recently [10]. As the injected current increases, we observe the regular PPO, irregular PPO, and chaotic state. To the best of our knowledge, this is the first observation of PPO, and the transition from PPO to chaos in a grating external-cavity feedback BAL system.

\section{EXPERIMENTAL SETUP AND RESULTS}

The experimental setup of the green external-cavity diode laser system is schematically shown in Fig. 1, and a detailed description of the setup has been given in Ref. [10]. The gain device in the diode laser system is a $1 \mathrm{~W}$ GaN broad-area diode laser around $515 \mathrm{~nm}$ (Nichia). An aspherical lens of $4.0 \mathrm{~mm}$ focal length is used to collimate the output beam from the BAL. The collimated beam is incident on a bulk reflective holographic grating with a groove density of 2400 lines/mm (Thorlabs, GH13-24U). The first-order diffracted beam from the grating is guided back into the diode laser as feedback beam. A half-wave plate is used to switch the polarization direction of the laser beam incident upon the grating. A beam splitter, BS1, (R 1\%) is inserted in the external cavity, and reflects part of the beam to a silicon PIN photodiode, PD1, (Melles Griot, 13DAH001); after a high-frequency amplifier (frequency below 1 $\mathrm{MHz}$ is blocked), the amplified electronic signal is sent to an electrical spectrum analyzer (Tektronix 2753P) to measure the intensity noise spectrum. The zeroth-order diffracted beam from the grating is the output of the laser system. The second beam splitter, BS2, reflects part of this beam to the second photodiode, PD2, (Thorlabs, DET210), and a 
1-GHz digital oscilloscope (LeCroy, WaveSurfer $104 \mathrm{Xs}$ ) is used to measure the time series of the generated electronic signal.

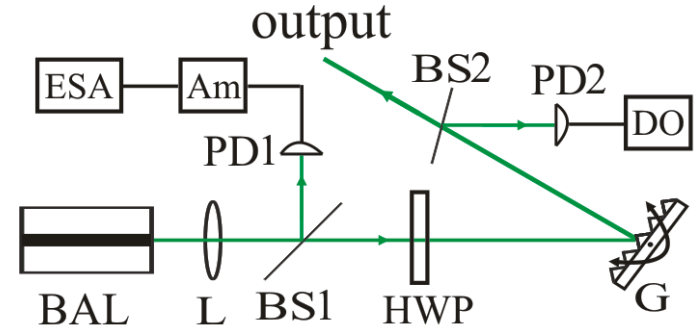

Fig. 1. Experimental setup of the Littrow external-cavity green diode laser system for dynamics investigation. BAL, broad-area diode laser; $\mathrm{L}$, lens; BS1 and BS2, beam splitters; HWP, half-wave plate; G, holographic grating; PD1 and PD2, photodiodes; DO, digital oscilloscope; Am, amplifier; ESA, electrical spectrum analyzer.
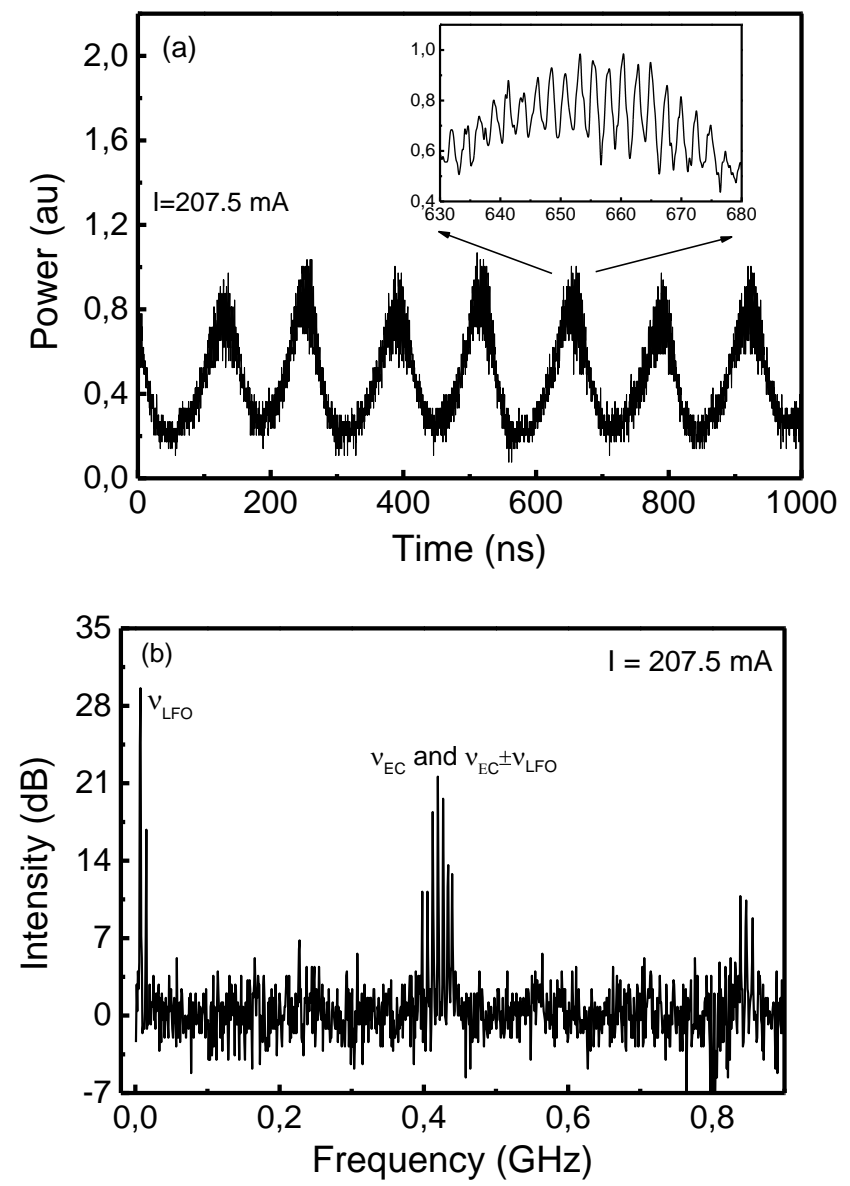

Fig. 2. Time series (a) and intensity noise spectrum (b) of output beam from the external-cavity green diode laser system with an injected current of $207.5 \mathrm{~mA}$. The inset in (a) shows a close up of the peak of one of the pulse packages.

In this paper, the green BAL system is operated in p-polarized mode, i.e., the laser beam is polarized perpendicular to the lines of the grating [10]. Thus the first-order diffraction efficiency of the grating is around $29 \%$ [10]. Assuming the coupling efficiency of the feedback beam into the laser cavity is 0.5 , the feedback strength is around $14.5 \%$, a moderate feedbackstrength for BALs [14,20]. The physical length of the external cavity is around $33 \mathrm{~cm}$.

The threshold current of the freely running BAL is around $250 \mathrm{~mA}$, and the slope efficiency is around 1.0 W/A; $1.1 \mathrm{~W}$ output power is obtained with an operating current of $1.4 \mathrm{~A}$. When the external-cavity diode laser system is operated in p-polarized mode, the threshold is decreased to $205.5 \mathrm{~mA}$, and the wavelength is around $511.9 \mathrm{~nm}$. In the experiment, we keep the feedback grating untouched, i.e., both the length of the external cavity and the wavelength of the green externalcavity laser system are unchanged when the injected current is adjusted.

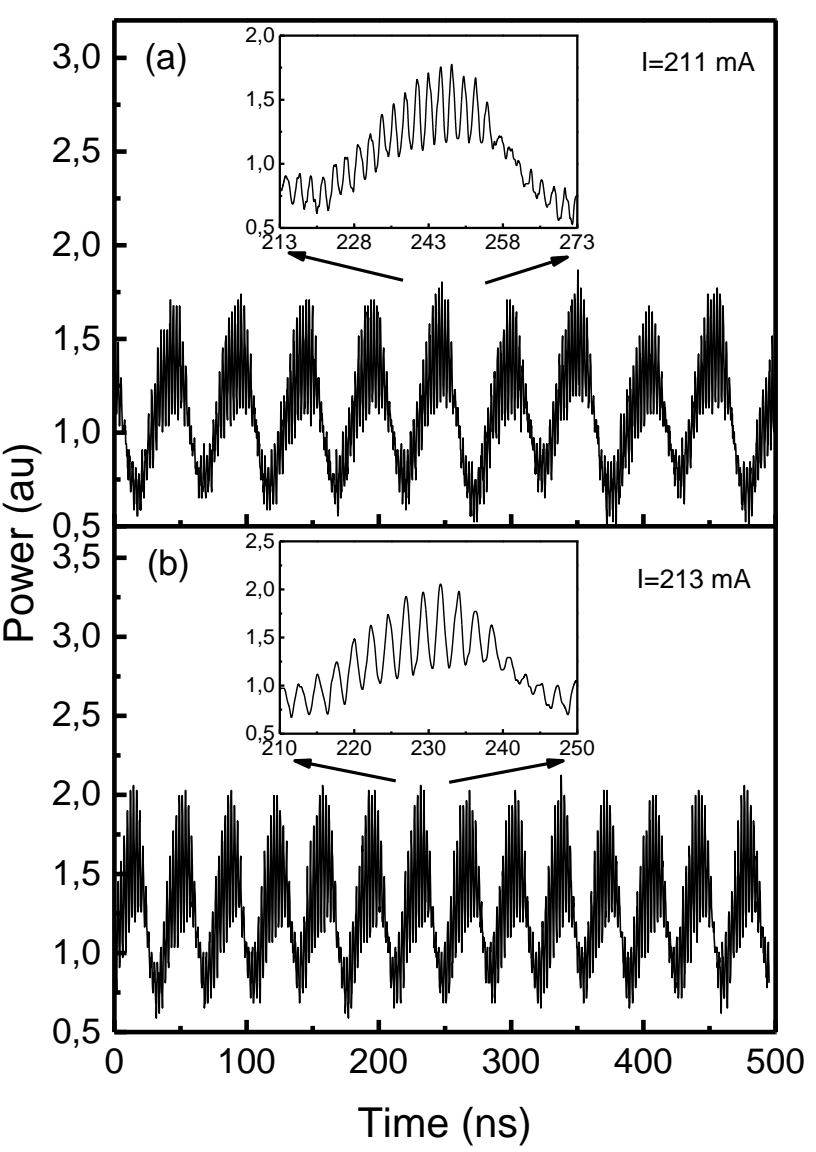

Fig. 3. Time series of the output beam from the external-cavity green diode laser system with an injected current of $211 \mathrm{~mA}$ (a) and $213 \mathrm{~mA}$ (b). The insets show a close up of one of the pulse packages.

Fig. 2(a) shows the time series of the output beam with an injected current of $207.5 \mathrm{~mA}$. A low-frequency periodic oscillation is observed with a frequency around 7.2 MHz. The inset of Fig. 2(a) shows the details of the time series in short time scale. An oscillation with a period around $2.4 \mathrm{~ns}$ is observed. Considering the physical length of the external-cavity and the thickness and refractive index of the lens, half-wave plate and beam splitter, the period of this high frequency oscillation is equal to the external-cavity delay time, i.e., $2 L / c$, where $c$ is the speed of light and $L$ is the optical external-cavity length. The high frequency oscillation is named as external-cavity oscillation with the single-round trip externalcavity frequency $v_{\mathrm{EC}}=c / 2 L,[13,20]$. Fig. 2 (b) shows the corresponding intensity noise spectrum of the output beam. Besides the peaks at the 
external-cavity oscillation frequency, $v_{\mathrm{EC}}$, a peak around 7.2 MHz and its harmonics for low frequency oscillation (LFO), $v_{\mathrm{LFO}}$, is also observed. Additionally, some peaks around $v_{\mathrm{EC}}$ with the frequency difference of $v_{\mathrm{LFO}}$ are also visible. Fig. (2) shows that the output of the green externalcavity diode laser manifests a typical dynamic state named regular PPO which mainly takes place in short-cavity feedback diode laser system $[12,13]$, where the external-cavity loop oscillation is modulated by a periodic LFO.

Fig. 3 shows the time series of the output beam of the diode laser system with injected currents of $211 \mathrm{~mA}$ and $213 \mathrm{~mA}$. The periodic LFO is observed for both cases. The oscillation frequencies are around 20 and $29 \mathrm{MHz}$ with the injected currents of $211 \mathrm{~mA}$ and $213 \mathrm{~mA}$, respectively. The insets in the figure show the details of one period of the regular LFOs, i.e., one of the pulse packages. The pulse packages, which consist of pulses occurring at the external-cavity round trip interval, are observed for both condition. This means the regular PPO takes place for both cases. Fig. 2 and Fig. 3 shows the oscillation frequency of the regular LFO, $\nu_{\mathrm{LFO}}$, increases with the increase of the injected current to the BAL. The increase of $v_{\mathrm{LFO}}$ with injected current was also observed in an integrated semiconductor laser operated in short cavity feedback regime [22]. The corresponding intensity noise spectra with these two injected currents are also measured, the results being similar to the noise spectrum shown in Fig. 2(b), and consistent with the time series shown in Fig. 3.
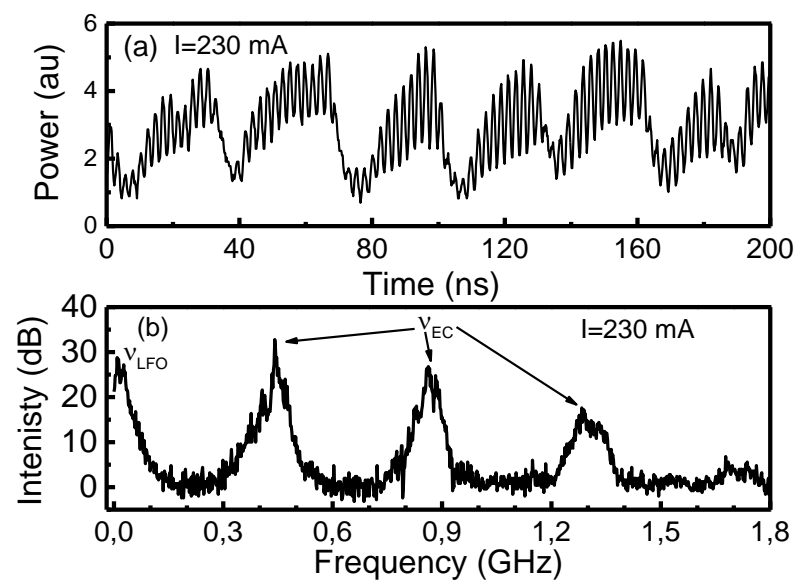

Fig. 4. Time series (a) and intensity noise spectrum (b) of the output beam from the external-cavity green diode laser system with an injected current of $230 \mathrm{~mA}$

When the injected current is further increased, the pulse packages oscillate irregularly. Fig. 4 shows the time series and the intensity noise spectrum of the output beam from the green external-cavity BAL system operated with injected current of $230 \mathrm{~mA}$. The time series still shows the pulse packages consisting of pulse train with the period of external-cavity delay time. However, the oscillation of the pulse packages is irregular, and the period of the pulse packages is decreased further, the average duration of the pulse packages shown in Fig. 4(a) is less than 30 ns. The corresponding intensity noise spectrum in Fig. 4(b) shows the broad peak for LFO at $v_{\mathrm{LFO}}$, and peaks for high frequency external-cavity oscillation at multiples of $\nu_{\mathrm{EC}}$. But the peaks at $\nu_{\mathrm{EC}} \pm \nu_{\mathrm{LFO}}$ originating from the mixing of external cavity loop frequency $v_{\mathrm{EC}}$ and LFO frequency $v_{\mathrm{LFO}}$, which is the indication of regular PPO, are not observed. All the peaks shown in Fig. 4(b) are much broader compared with the intensity noise spectrum shown in Fig. 2(b). This means that the intensity noise is increased strongly, and the dynamic behavior of the laser system is more complex. This is consistent with the time series of irregular PPO shown in Fig. 4 (a).

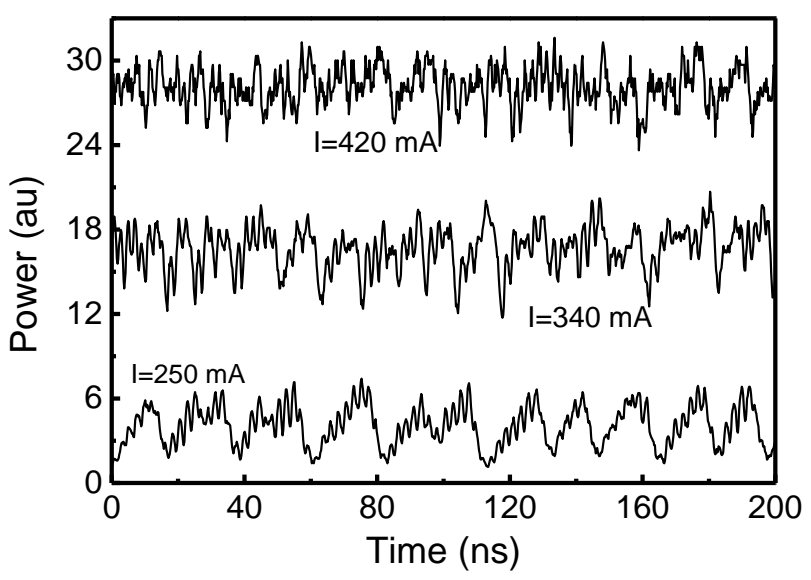

Fig. 5. Time series of the output beam from the external-cavity green diode laser system with different injected currents.

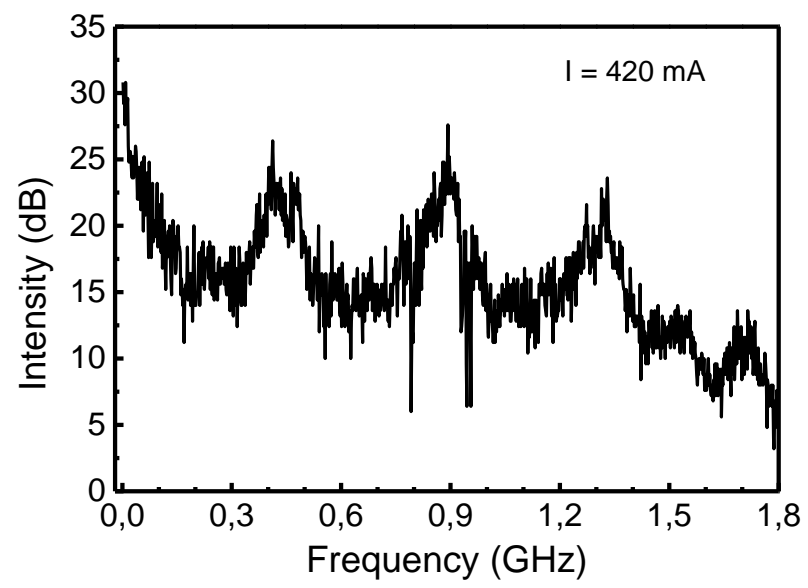

Fig. 6. Intensity noise spectrum of the output beam from the externalcavity green diode laser system with an injected current of $420 \mathrm{~mA}$.

We increase the injected current to $420 \mathrm{~mA}$, i.e., more than twice the threshold of the BAL system, and measure the time series and the intensity noise spectra of the output of the laser system. Fig. 5 shows the time series with injected currents of 250,340, and $420 \mathrm{~mA}$. When the injected current is $250 \mathrm{~mA}$, the time series shows irregular PPO, the frequency component of the high frequency external-cavity oscillation is not as clear as in the cases with lower injected currents. The average duration of the pulse package is less than $20 \mathrm{~ns}$, meaning that the oscillation of the pulse package is faster compared with the cases with lower injected currents. The pulse package is notclear when the injected current is increased to $340 \mathrm{~mA}$, and the pulses occurring at the externalcavity round trip interval is almost invisible. Finally, the time series shows a chaotic behavior with an injected current of $420 \mathrm{~mA}$. The corresponding intensity noise spectrum of the output beam with the injected current of $420 \mathrm{~mA}$ is shown in Fig. 6. The broad intensity noise spectrum from low frequency to high frequency indicates typical features of chaotic dynamics of the output beam. This is consistent with the chaotic time series shown in Fig. 5. 


\section{DISCUSSION}

The fast dynamic behavior is seldom investigated in a BAL with grating feedback. Pan et al. studied the dynamics of a BAL with grating feedback [21] by measuring the time series at different positions at the near field. When the injected current was low, a period-one oscillation was observed, and when the injected current was higher than 1.5 times threshold, chaotic dynamics was observed. Here we observe regular PPO in the green external-cavity diode laser system with injected currents near the threshold and an increase of oscillation frequency of the pulse packages with injected current.

Regular PPO was first observed by Heil et al. in 2001 in externalcavity feedback narrow strip diode laser systems [23]. The origin of PPO is the state of the laser wanders among the external-cavity modes and external-cavity anti-modes repeatedly, a result of saddle-node instability caused by external feedback [23,24]. This phenomenon mainly takes place in diode lasers with short-cavity feedback [12,13], i.e., when the relaxation oscillation frequency, $\nu_{\mathrm{RO}}$, of the solitary diode laser is lower than $v_{\mathrm{EC}}$. In practice, a length of the external-cavity around a few centimeters or less means short cavity feedback. Recently, we observed regular PPO in a BAL with a lateral-mode-selected long-cavity feedback [20]. Here, regular PPO is observed in a BAL with grating external-cavity feedback for the first time to our knowledge.

In the regular PPO condition, both the experimental and theoretical results showed the increase of LFO frequency $v_{\mathrm{LFO}}$ with injected current $[22,24]$, similar results are also observed in our experiment. When the injected current is increased further, the pulse packages first oscillate irregularly, and finally change to a chaotic state. The chaotic state with high injected current is similar to the observation in Ref. 21. Different routes to chaos in different diode laser systems have been found, such as period-doubling route to chaos in narrow-stripe single mode diode laser [25], period-doubling and quasiperiodic route to chaos in spinpolarized vertical-cavity surface-emitting laser [26]. Here, we observe the transition from regular PPO to chaos in a BAL with grating external feedback for the first time to our knowledge.

Normally, the LFO frequency of regular PPO is in the range of a few hundreds of $\mathrm{MHz}$ to a $\mathrm{GHz}[12,13,20,22,23]$. Our experiment on the green external-cavity BAL system shows the LFO frequency is in the range from a few $\mathrm{MHz}$ to a few tens of $\mathrm{MHz}$, much lower than the previous results. There may be two reasons for the slow LFO, since the length of the external-cavity here is around 10 times of cavity length for short cavity condition, the external-cavity oscillation period in a pulse package, i.e., $2 L / c$, is also around 10 times of the oscillation period of short cavity. The second reason is, due to the long external cavity, more external-cavity modes can be excited, the laser system may visit more external-cavity modes during one PPO cycle $[23,24]$.

Depending on the length of external cavity, the external-cavity feedback for diode laser system is classified into two regimes: short and long cavity regimes. When the relaxation oscillation frequency of the solitary diode laser, $\nu_{\mathrm{RO}}$, is lower than $\nu_{\mathrm{EC}}$, the feedback is in short cavity regime; otherwise, it is in long cavity regime [22-24]. With moderate feedback strength, the PPO mainly takes place in diode laser with short cavity feedback, while LFF and chaos take place in long cavity regime. The transition from the short to long cavity regime can be achieved by increasing either the length of external cavity [13] or the injected current [22]. Normally, an external-cavity length of a few centimeters means short cavity feedback, in this point, the $33 \mathrm{~cm}$ external-cavity length in our case is in the long cavity regime. The $\nu_{\mathrm{RO}}$ of the solitary diode laser is proportional to the square root of the difference between the injected current and the solitary laser threshold current [22], means the definition of $\nu_{\mathrm{RO}}$ is valid only when the injected current is above the threshold current. Thus, the classification of short and long cavity feedback is meaningful only when the injected current is higher than the threshold of the solitary diode laser. In our case, the PPO mainly takes place with the injected current below the threshold of the solitary laser. In this point, this is a new regime for the external-cavity diode laser system. Theoretical research on the dynamics of diode laser with external-cavity feedback in this regime, i.e., below the threshold of solitary laser, is undergoing.

\section{CONCLUSION}

In conclusion, the dynamic behavior of a high-power green BAL with grating external-cavity feedback is investigated. As the injected current is increased, different dynamic behaviors from regular PPO, irregular PPO to broadband chaos are observed. It is the first time that regular PPO and the transition from regular PPO to chaos has been observed in a grating external-cavity BAL system.

Funding information. Danish Energy Technology Development and Demonstration Program (EUDP) (64014-0171)

\section{References}

1. T. Miyoshi, S. Masui, T. Okada, T. Yanamoto, T. Kozaki, S. Nagahama, and T. Mukai, "510-515 nm InGaN-based green laser diodes on c-plane GaN substrate," Appl. Phys. Express 2, 062201 (2009).

2. A. Avramescu, T. Lermer, J. Müller, S. Tautz, D. Queren, S. Lutgen, and U. Strau $\beta$, "InGaN laser diodes with $50 \mathrm{~mW}$ output power emitting at 515 nm," Appl. Phys. Lett. 95, 071103 (2009).

3. http://www.nichia.co.jp

4. J. Hofmann, G. Blume, D. Jedrzejczyk, B. Eppich, D. Feise, S. Kreutzmann, A. Sahm, and K. Paschke, "Miniaturized diode laser module emitting green light at $532 \mathrm{~nm}$ with a power of more than $900 \mathrm{~mW}$ for next-generation holographic displays," Opt. Rev. 23,141-145 (2016).

5. A. Müller, S. Marschall, O. B. Jensen, J. Fricke, H. Wenzel, B. Sumpf, and P. E. Andersen, "Diode laser based light sources for biomedical applications," Laser Photonics Rev. 7, 605-627 (2013).

6. J. F. Sell, W. Miller, D. Wright, B. V. Zhdanov, and R. J. Knize, "Frequency narrowing of a $25 \mathrm{~W}$ broad area diode laser," Appl. Phys. Lett. 94, 051115 (2009).

7. M. Chi, G. Erbert, B. Sumpf, and P. M. Petersen, "Tunable high-power narrow-spectrum external-cavity diode laser based on tapered amplifier at 668 nm," Opt. Lett. 35, 1545-1457 (2010).

8. N. Ruhnke, A. Müller, B. Eppich, M. Maiwald, B. Sumpf, G. Erbert, and G. Tränkle, "400 mW external cavity diode laser with narrowband emission at 445 nm," Opt. Lett. 39, 3794-3797 (2014).

9. M. Chi, O. B. Jensen, and P. M. Petersen, "Tuning range and output power optimization of an external-cavity GaN diode laser at $455 \mathrm{~nm}$," Appl. Opt. 55, 2263-2269 (2016).

10. M. Chi, O. B. Jensen, and P. M. Petersen, "Green high-power tunable external-cavity GaN diode laser at 515 nm," Opt. Lett. 41, 4154-4157 (2016).

11.S. K. Mandre, I. Fischer, and W. Elsässer, "Control of the spatiotemporal emission of a broad-area semiconductor laser by spatially filtered feedback," Opt. Lett. 28, 1135-1137 (2003).

12.S. K. Mandre, I. Fischer, and W. Elsässer, "Spatiotemporal emission dynamics of a broad-area semiconductor laser in an external cavity: stabilization and feedback-induced instabilities," Opt. Commun. 244, 355365 (2005).

13. A. Takeda, R. Shogenji, and J. Ohtsubo, "Dynamics and pulse-package oscillations in broad-area semiconductor lasers with short optical feedback," Appl. Phys. Lett. 101, 231105 (2012).

14.J. S. Lawrence and D. M. Kane, "Broad-area diode lasers with plane-mirror and phase-conjugate feedback," J. Lightwave Technol. 20, 100-104 (2002).

15. Y. Fujita and J. Ohtsubo, "Optical-feedback-induced stability and instability in broad-area semiconductor lasers," Appl. Phys. Lett. 87, 031112 (2005). 
16. M. Arahata and A. Uchida, "Inphase and antiphase dynamics of spatiallyresolved light intensities emitted by a chaotic broad-area semiconductor laser," IEEE J. Sel. Topics Quantum Electron. 21, 1800609 (2015).

17. P. P. Vasil'ev and I. H. White, "Phase-conjugation broad area twin-contact semiconductor laser," Appl. Phys. Lett. 71, 40-42 (1997).

18. T. Tachikawa, R. Shogenji, and J. Ohtsubo, "Observation of multi-path interference in broad-area semiconductor lasers with optical feedback," Opt. Rev. 16, 533-539 (2009).

19. S. Wolff, A. Rodionov, V. E. Sherstobitov, C. Doering, H. Fouckhardt, "Selfpulsation in broad-area lasers with transverse-mode selective feedback," Opt. Commun. 265, 642-648 (2006).

20. M. Chi and P. M. Petersen, "Dynamics of a broad-area diode laser with lateral-mode-selected long-cavity feedback," J. Appl. Phys. 116, 103101 (2014)

21. M. Pan, D. J. Evans, G. R. Gray, L. M. Smith, R. E. Benner, C. W. Johnson, and D. D. Knowlton, "Spatial and temporal coherence of broad-area lasers with grating feedback," J. Opt. Soc. Am. B 15, 2531-2536 (1998).

22.J. P. Toomey, D. M. Kane, C. McMahon, A. Argyris, and D. Syvridis, "Integrated semiconductor laser with optical feedback: transition from short to long cavity regime," Opt. Express 23, 18754-18762 (2015).

23.T. Heil, I. Fischer, W. Elsäßer, and A. Gavrielides, "Dynamics of semiconductor lasers subject to delayed optical feedback: the short cavity regime," Phys. Rev. Lett. 87, 243901 (2001).

24. T. Heil, I. Fischer, W. Elsäßer, B. Krauskopf, K. Green and A. Gavrielides, "Delay dynamics of semiconductor lasers with short external cavities: Bifurcation scenarios and mechanisms," Phys. Rev. E 67, 066214 (2003).

25. J. Ye, H. Li, and J. G. Mclnerney, "Period-doubling route to chaos in a semiconductor laser with weak optical feedback," Phys. Rev. A 47, 22492252 (1993)

26. N. Li, H. Susanto, B. R. Cemlyn, I. D. Henning, and M. J. Adams, "Stability and bifurcation analysis of spin-polarized vertical-cavity surface-emitting lasers," Phys. Rev. A 96, 013840 (2017). 